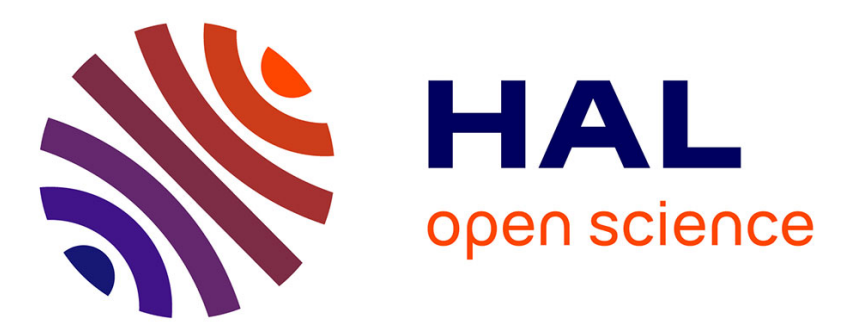

\title{
A numerical framework to predict the fatigue life of lattice structures built by additive manufacturing
} Alexis Burr, Théo Persenot, Pierre-Thomas Doutre, Jean-Yves Buffiere, Pierre Lhuissier, Guilhem Martin, Rémy Dendievel

\section{- To cite this version:}

Alexis Burr, Théo Persenot, Pierre-Thomas Doutre, Jean-Yves Buffiere, Pierre Lhuissier, et al.. A numerical framework to predict the fatigue life of lattice structures built by additive manufacturing. International Journal of Fatigue, 2020, 139, pp.105769. 10.1016/j.ijfatigue.2020.105769 hal-02952483

\section{HAL Id: hal-02952483 \\ https://hal.science/hal-02952483}

Submitted on 29 Sep 2020

HAL is a multi-disciplinary open access archive for the deposit and dissemination of scientific research documents, whether they are published or not. The documents may come from teaching and research institutions in France or abroad, or from public or private research centers.
L'archive ouverte pluridisciplinaire HAL, est destinée au dépôt et à la diffusion de documents scientifiques de niveau recherche, publiés ou non, émanant des établissements d'enseignement et de recherche français ou étrangers, des laboratoires publics ou privés. 


\section{Graphical Abstract}

A numerical framework to predict the fatigue life of lattice structures built by additive manufacturing

Alexis Burr, Théo Persenot, Pierre-Thomas Doutre, Jean-Yves Buffiere, Pierre Lhuissier, Guilhem Martin, Rémy Dendievel

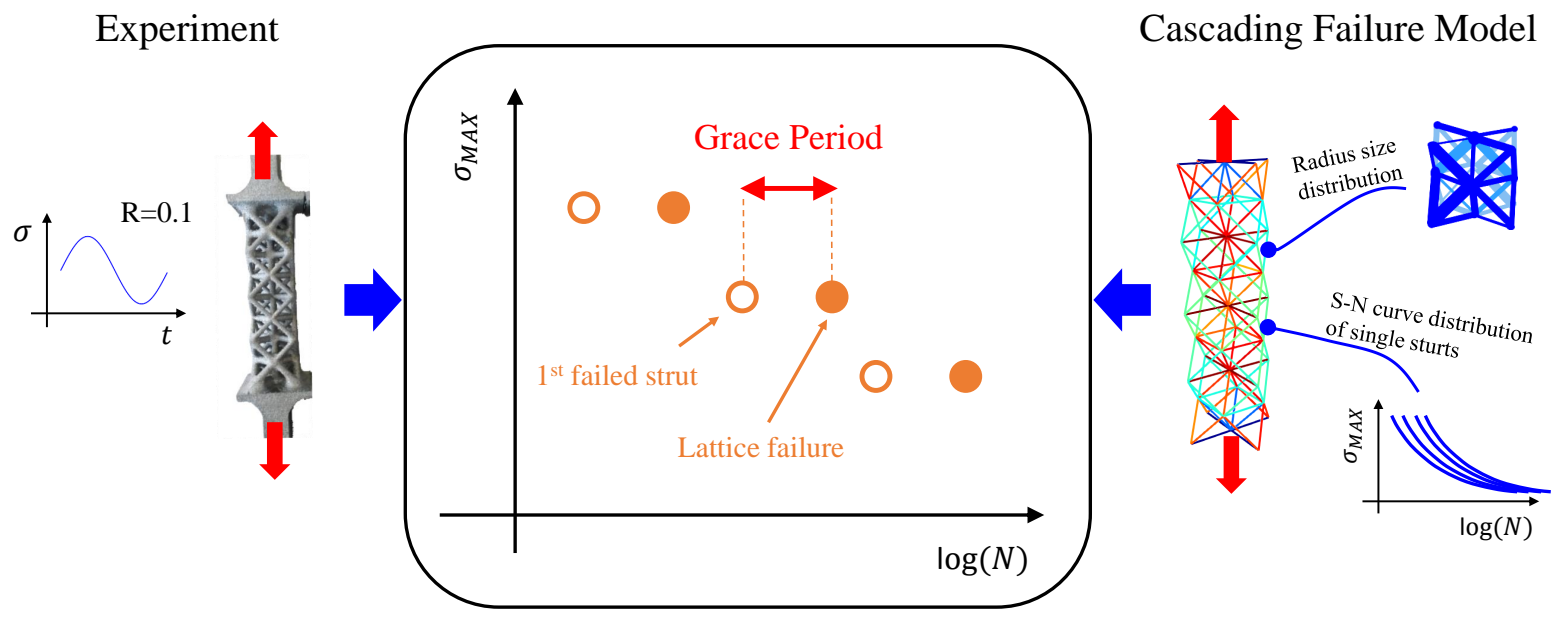




\section{Highlights}

A numerical framework to predict the fatigue life of lattice structures built by additive manufacturing

Alexis Burr, Théo Persenot, Pierre-Thomas Doutre, Jean-Yves Buffiere, Pierre Lhuissier, Guilhem Martin, Rémy Dendievel

- Fatigue failure of lattice structures is gradual exhibiting a so-called grace period

- Fatigue properties of lattices depend on additive manufacturing inherited defects

- A cascading failure-based model correctly predicts grace period and fatigue life 


\title{
A numerical framework to predict the fatigue life of lattice structures built by additive manufacturing
}

\author{
Alexis Burr ${ }^{\mathrm{a}, *}$, Théo Persenot ${ }^{\mathrm{b}}$, Pierre-Thomas Doutre ${ }^{\mathrm{c}}$, Jean-Yves Buffiere $^{\mathrm{b}}$, Pierre Lhuissier $^{\mathrm{a}}$, Guilhem \\ Martin ${ }^{\mathrm{a}}$, Rémy Dendievel ${ }^{\mathrm{a}, *}$ \\ ${ }^{a}$ Univ. Grenoble Alpes, CNRS, Grenoble INP ${ }^{1}$, SIMaP, F-38000 Grenoble, France \\ ${ }^{b}$ INSA Lyon, CNRS, MATEIS, F-69621 Villeurbanne, France \\ ${ }^{c}$ Univ. Grenoble Alpes, CNRS, Grenoble INP ${ }^{1}$, G-SCOP, F-38000 Grenoble, France
}

\begin{abstract}
The fatigue properties of lattice structures produced by S-EBM are investigated. Fatigue failure is shown to be gradual, fostering the concept of grace period, defined as the number of cycles lived by the lattice structure after the failure of the first strut. A numerical framework based on the cascading failure of struts is proposed, relying on a damage accumulation law. Each strut is assigned a radius as well as an S-N curve, which both depend on the manufacturing conditions. Through statistical analyses, we demonstrate that the model can correctly predict the grace period and the fatigue life of experimental specimens.
\end{abstract}

Keywords: Lattice structures, S-N curves, cumulative damage, simulation (numerical, experimental), additive manufacturing

\section{Introduction}

Architectured materials such as lattice structures have received a great interest over the last few years, in particular with the emergence of additive manufacturing (AM) $[1,2,3]$. These materials exhibit interesting properties, e.g. large strength-to-weight ratio that can be a key driver for biomedical or aerospace applications. Properties of lattice structures can be tailored by changing the topology or varying the relative density. However, important challenges are still to be overcome. For instance, materials produced by powder-bed fusion technologies such as laser beam melting (LBM) and electron beam melting (EBM) still suffer from the presence of defects inherited from the process, in particular from the rough surface. It is particularly true in the case of fatigue, where this poor surface quality leads to a significant knock-down of the fatigue performances of as-built parts in comparison with machined ones (see e.g. $[4,5,6,7,8]$ ). However, most studies on materials produced by additive manufacturing have investigated the fatigue performances of machined samples (e.g., $[9,10])$. Their results cannot be directly used for lattice structures as the latter cannot be machined.

Studies on lattice structures focus primarily on which parameters (e.g., relative density, the topology of the unit cell, strut diameter ...) must be tuned to give the best or most suited effective properties. In the

\footnotetext{
${ }^{*}$ Corresponding authors.

Email addresses: alexis.burr@protonmail.com (Alexis Burr), remy.dendievel@simap.grenoble-inp.fr (Rémy Dendievel)

${ }^{1}$ Institute of Engineering Univ. Grenoble Alpes
} 
case of static (e.g., $[11,12,13])$ or fatigue behavior (e.g., $[14,15,16])$ of metal parts made by LBM or EBM, studies dedicated to lattice structures investigate the effects of topologies or relative density [17, 18]. Authors usually evaluate experimentally the effect of lattice parameters on mechanical properties (stiffness, strength, energy absorption ...) and select between stretching and bending-dominated structures for the desired set of requirements. As far as fatigue is concerned, most studies investigate the compression - compression mode. Performing tensile fatigue tests is more difficult because it requires some geometrical adjustments to clamp specimens, to limit stress concentration near junctions and also to allow failure localization in a gauge length. Only a few topologies have been manufactured so far [19, 20, 21], mainly by LBM. Recently, Lietaert et al. [22] published the first comprehensive study on the fatigue behavior of a Ti-6Al-4V lattice structures made by LBM with different stress ratios : $R=-1$ (tension-compression), $R=10$ (compressioncompression) and $R=0.1$ (tension-tension). Most of the time, authors study the final failure of lattice structures, without drawing a particular interest to the cascading failure of single struts. The objective is to determine the effective fatigue properties (number of cycles to final failure) as a function of relative density, topology, and constitutive materials. Such a strategy often requires trial-and-error. Thus, there is a need to be able to design an optimized lattice structure for a given application, by limiting these time-consuming trial-and-errors. Numerical methods are thus required to predict the fatigue life of these structures.

From the modeling point of view, authors generally perform a straightforward discretization of lattice structures into beam elements. Two strategies arise from the literature. Some authors [23, 22] use the beam theory to calculate local stress in struts according to the applied macroscopic stress on the lattice structure. Then, they derive an S-N curve for the single struts of which lattices are made of. Conversely, another strategy consists of using the S-N curve of single struts to predict the fatigue life as well as the chronology of local failure events occurring in lattice structures. This implies to take into account the progressive stress redistribution occurring in unbroken struts. This also requires to quantify the local damage in each strut during the whole cyclic process. Taking into account this damage accumulation is challenging. A few authors have attempted to do so (see e.g., [24, 25, 26, 27]). They relied on a damage accumulation law based on the Miner's rule [28], consisting in adding up the damage of struts at the different stress levels they were submitted to. A unique S-N curve of the constitutive material was considered for the fatigue behavior at the scale of individual struts. This means that every strut had exactly the same fatigue properties, although experimental fatigue results on single struts are usually scattered $[4,29,30,10,7]$.

The present work focuses on the sensitivity of fatigue life predictions for lattice structures to two independent sources of variability: (i) radius size distribution and (ii) scattered S-N curves. To that end, we will use two kinds of experimental results obtained on EBM. Previous results obtained at the scale of single struts $[7,31]$ will be introduced as input data of the model. New experimental results at the scale of the lattice structure will be used for validating the predictions at the macro-scale. The different sources of variability will be explicitly taken into account and their effects on fatigue life and cascading failure event will be discussed.

In section 2, our cascading failure-based model used for fatigue life predictions is described in details. Section 3 proposes specific case studies for which experimental results and numerical predictions will be 
compared. A first application of the model will concern precisely the as-built experimental case. Extensions will be presented, taking into account different kinds of variability (geometry and/or single strut S-N curve). In section 4 , limits, and assumptions of the model are discussed.

\section{Cascading failure-based model}

In this section, we provide a detailed description of the developed cascading failure-based model which aims at predicting the fatigue life of lattice structures, based on the fatigue performances determined experimentally from the constitutive single struts.

\subsection{Inputs}

To demonstrate the versatility of our approach, we give hereafter an overview of the various inputs that are required by the model.

- INPUT 1: lattice unit cell. A lattice structure is defined as an assembly of nodes connected by struts. Two examples of lattice unit cells are given in Fig. 1a but any lattice structure can be used as input. Several types of struts (here types A, B, and C) can be defined.

- INPUT 2: struts radius size distribution for each type of strut (here A, B, and C). As illustrated in Fig. 1a-b, it means that a given strut can have a given radius. This is justified by a previous study [7], that showed that struts having different orientations with respect to the building direction exhibit different geometrical characteristics: radius, roughness, morphology.

- INPUT 3: a set of S-N curves allowing to assign a given S-N curve to a given strut (see Fig. 1c). This offers the opportunity to have struts with varying fatigue properties. This is motivated by the fact that experimental S-N curves are often scattered, in particular when dealing with AM parts with poor surface quality. In practice, it consists of a distribution of N-values at fixed stress for each type of struts (here A, B, and C). The S-N curves are then provided by a classical Basquin law (detailed in section 3.3.2).

\subsection{Algorithm}

In the following, the stress will always refer to the maximum stress of a cycle denoted $\sigma_{\text {max }}$, which is the one used in the S-N curve of single struts. Local stresses in a strut are calculated by classical finite element calculations using Timoshenko beam elements with isotropic elasticity.

The model simulates the successive fatigue failure of individual struts constituting the lattice structure. It strongly relies on the concept of damage. As illustrated in Fig. 2a, the damage $D$ occurring at given stress is defined as the ratio of the number of cycles undergone $N$ over the number of cycles to failure $N^{\max }$ :

$$
D=\frac{N}{N^{\max }}=1-\frac{n^{r}}{N^{\max }},
$$


(a)
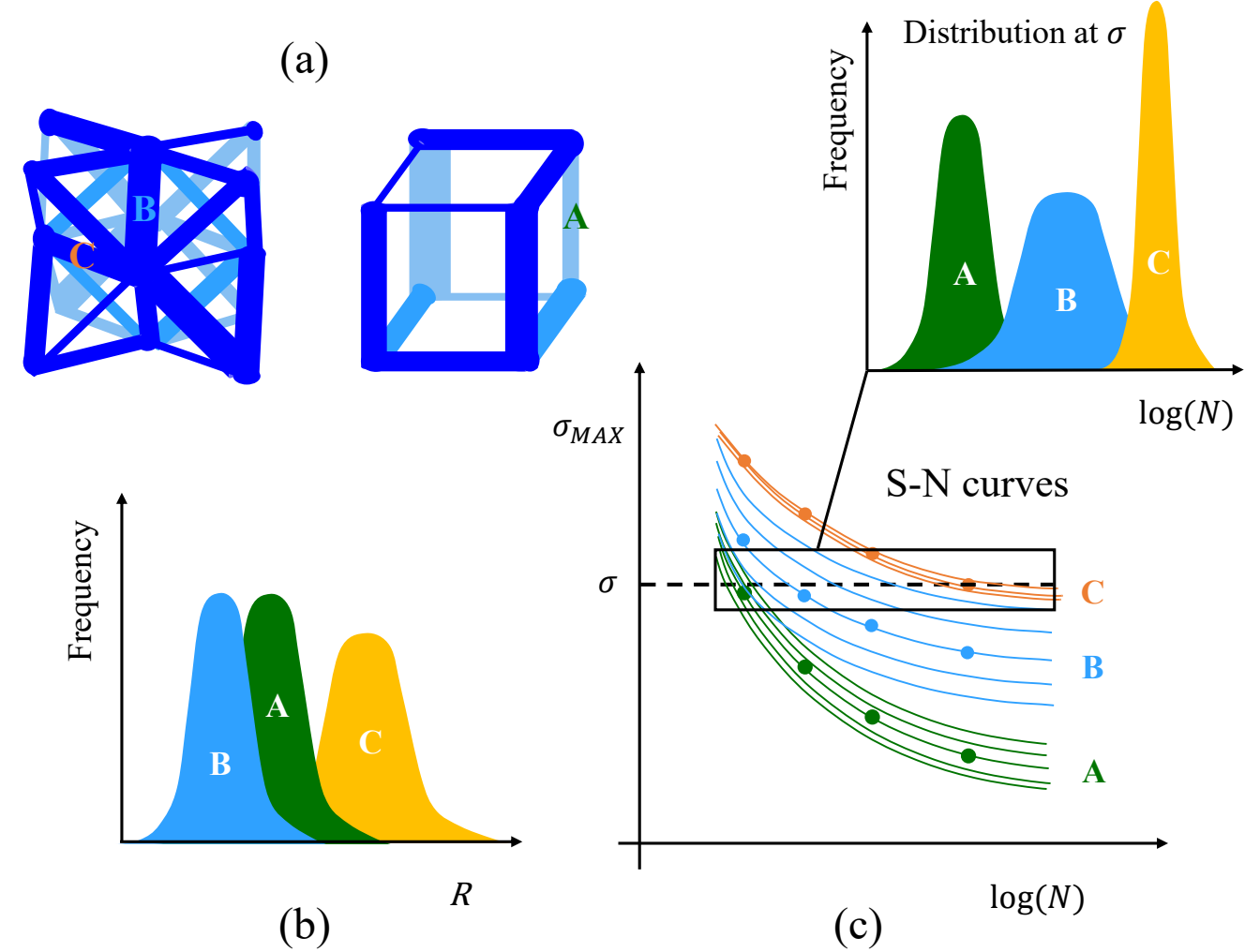

Figure 1: Overview of the different inputs of the model. (a) Examples of lattice unit cells cubic and octet-truss with varying strut radii. The proposed algorithm is independent of the chosen unit cell. (b) Size distributions for radius $R$ for types A, B, and $\mathrm{C}$. This means every strut can have a given radius. (c) S-N curves for three different types of struts A, B, and C from (a). Dots stand for the mid-range fatigue curve (failure probability of 50\%). A different S-N curve, i.e. fatigue behavior can be assigned to every strut within the lattice leading to a distribution of $N$ for every type of strut at a given stress. 


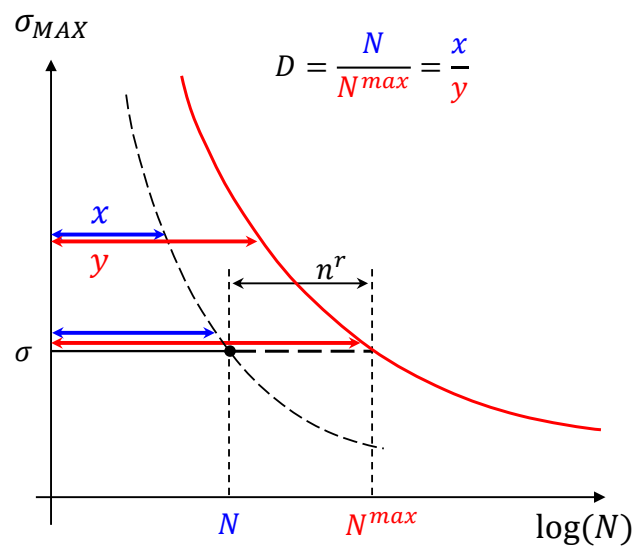

(a)

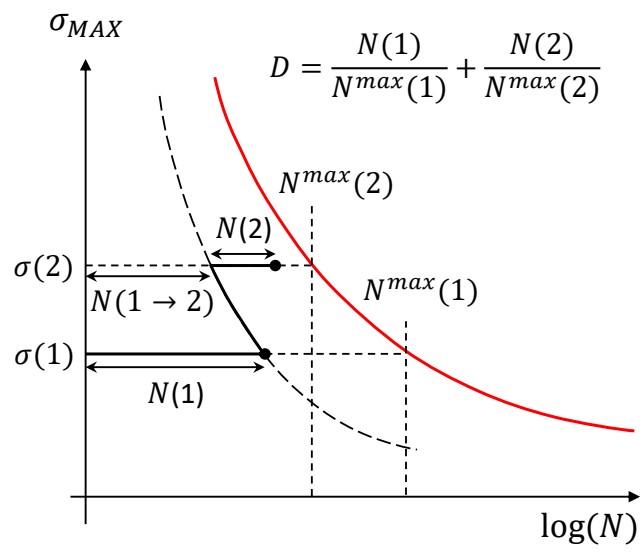

(b)

Figure 2: Schematic of S-N curves. (a) Iso-damage curve (dashed line) using the definition of the damage (Eq. 1) as the ratio of number of cycles $N$ undergone at stress $\sigma$ over the maximum number of cycles possible $N^{\text {max }}$ (i.e. the fatigue life, red line). $n^{r}$ is defined as the remaining life. (b) Concept of damage accumulation illustrated for two stress levels $\sigma(1)$ and $\sigma(2)$ (Eq. 2). $N(1 \rightarrow 2)$ is the equivalent number of cycles at stress $\sigma(2)$ which corresponds to the damage $D(1)=\frac{N(1)}{N^{m a x}(1)}$ experienced at stress $\sigma(1)$.

where $n^{r}$ is the remaining life of the strut at this given stress. Thus the S-N curve can be used to define for any stress value, an equivalent number of cycles sustained by the strut corresponding to this degree of damage, see Fig. 2a and the representation of the so-called "iso-damage" curve. If a strut experiences various stress levels throughout its lifetime, the Miner's rule [28] is applied and damage, calculated at each of these stress levels, can be summed. Eq. 2 is a generalization of Eq. 1 for a strut submitted to $i$ levels of stress :

$$
D=\sum_{k=1}^{i} \frac{N(k)}{N^{\max }(k)} .
$$

Fig. $2 \mathrm{~b}$ is a schematic that aims at clarifying this rule in the case of a strut experiencing first a stress level $\sigma(1)$ during $N(1)$ cycles, followed by a stress level $\sigma(2)$ during $N(2)$ cycles. Conditions to break a strut under cyclic loading are met once $D=1$.

In the present case, an iterative procedure is performed. An iteration (a step) consists in :

(i) deriving the stress distribution in every strut, using a finite element calculation based on Timoshenko beams;

(ii) identifying the strut to break (i.e. the one with the smallest remaining life).

Note that, in such a frame, constant stress is used during the FE calculations. The value of this stress is the maximum value of the cyclic loading. We do not take into account the effect of an initiating and propagating crack at the level of a single strut. As a result, the damaging of struts is a discontinuous process that is only updated once a strut has failed, i.e. has reached its fatigue life for a given maximum stress. The developed algorithm relies on the same idea proposed by Demiray et al. and Zargarian et al. in [24, 26, 27], whereas 
Hedayati et al. [25] suggested a more continuous damaging process by running simulations after an increment of cycles $(\Delta N)$ that evolves depending on failure events.

Let us introduce the following notations with index $i$ referring to step $i$ and index $j$ referring to strut j. $\sigma_{j}(i)$ is the stress in strut $j$ at step $i . N_{j}(i)$ is the number of cycles undergone by strut $j$ at step $i$, and $N_{j}^{\max }(i)$ its fatigue life, i.e. the maximum number of cycles that strut $j$ could sustain at stress $\sigma_{j}(i)$. According to Eq. 1, the damage undergone in strut $j$ during step $i$ can be calculated as follows :

$$
D_{j}(i)=\frac{N_{j}(i)}{N_{j}^{\text {max }}(i)} .
$$

Thus at step $i$ any unbroken strut $j$ meets the following conditions:

$$
D_{j}=\sum_{k=1}^{i} D_{j}(k) \leq 1,
$$

where $D_{j}$ is the cumulative damage in strut $j$. Using these definitions, the algorithm can be summarized as follows for a step $i$.

1. Run finite element simulation for step $i$ that provides the stress distribution in struts within the lattice structure.

2. The number of cycles undergone by every strut is calculated using the Miner's rule based on the accumulated damage (eq. 3 and see Fig. 2b extrapolated to steps $i-1$ and $i$ ):

$$
D_{j}(i-1)=\frac{N_{j}(i-1)}{N_{j}^{\max }(i-1)}=\frac{N_{j}(i-1 \rightarrow i)}{N_{j}^{\max }(i)}
$$

For strut $j$, the equivalent fatigue life $N_{j}(i-1 \rightarrow i)$ is thus:

$$
N_{j}(i-1 \rightarrow i)=D_{j}(i-1) N_{j}^{\max }(i) .
$$

3. The remaining life denoted $n_{j}^{r}(i)$ is therefore calculated for each strut as follows:

$$
n_{j}^{r}(i)=N_{j}^{\max }(i)\left(1-\sum_{k=1}^{i-1} D_{j}(k)\right)
$$

Note that for step $i=1$, damage is equal to zero for all struts, thus $n_{j}^{r}(1)=N_{j}^{\max }(1)$ (see Fig. 2b).

4. The duration of step $i$ is defined as the number of cycles between the failure of the $(i-1)$-th strut and the $i$-th strut. It is the strut with the smallest remaining life $n_{j}^{r}(i)$ at step $i$ which is removed for the subsequent computations. Therefore, the number of cycles $n(i)$ sustained by every strut within the lattice structure during step $i$ has no reason to be constant. This number is equal to:

$$
n(i)=\min _{j}\left\{n_{j}^{r}(i)\right\}
$$

5. Damage of every strut is updated according to the additional number of cycles $n(i)$ they have lived and the total number of cycles undergone by the lattice structure can be written as follows :

$$
N=\sum_{k=1}^{i} n(k) .
$$


6. The condition to stop the simulation can be arbitrarily chosen. It can be defined by an important increase of strain, a given percentage of failed struts, or when the fatigue life is converging as in the case of the present work, i.e. removing new struts barely changes the predicted fatigue life. If the chosen condition is not fulfilled, then the iterative process is repeated. In case the condition is met, then the iterative process is stopped and the predicted fatigue life is $N$.

\subsection{Stress calculation}

In the previous procedure, one has to define local stresses at the scale of the struts, as well as the macroscopic stress at the scale of the lattice. A stretching-dominated lattice structure is used. Therefore, in a first approximation, the local stress in each strut is the normal force divided by the cross-section of the strut. In fact, for strut $j$ at step $i$, the stress is computed as written in Eq. 10 :

$$
\sigma_{j}(i)=\lambda \frac{F_{j}(i)}{S_{j}}
$$

with $F_{j}(i)$ the normal force, $S_{j}$ the cross-section of strut $j$ and $\lambda$ a corrective coefficient. This coefficient aims at bypassing a full 3D-FEM calculation. The introduction of such a corrective coefficient allows different effects to be taken into account: the section variability inherited from the AM process AM process (see for example $[32,33])$, the presence of rigid nodes instead of pin joints, and the presence of bending moments near nodes. It was decided to give $\lambda$ a unique value for a given condition over the calculations (as-built or chemically etched). This unique value was identified so that the numerical predicted fatigue life of the first strut to fail into the lattice structure, matches the experimental fatigue life given by the Basquin law for single struts (see Eq. 12). In fact, the previously mentioned parameters (i.e., section variability, stress concentration factors, bending moments) are different whether as-built or etched struts are considered. In particular, etching significantly modifies the local surface aspect near nodes, thus the intensity of stress raisers. As a result, two different values of the corrective coefficient $\lambda$ were used: a first one set to 1.47 for as-built specimens, and a second one set to 1.62 for etched specimens.

The macroscopic stress $\sigma$ applied on the lattice structure is defined by the ratio of the applied macroscopic force $F_{\text {macro }}$ over the section of the numerical lattice structure $S_{\text {lattice }}: \sigma=\frac{F_{\text {macro }}}{S_{\text {lattice }}}$ as illustrated in Fig. $3 \mathrm{~b}$.

\subsection{Outputs}

Numerous outputs can be extracted from this model such as the macroscopic stress tensor or the stress distribution within the lattice structure. Since this work is focused on the fatigue life prediction, we can also introduce the "grace period" (GP) as the number of cycles lived by the lattice structure after the failure of the first strut. The "grace ratio" (GR) is defined as the ratio of this grace period over the fatigue life of the whole structure (expressed as a percentage). Combining equations 8 and 9, the grace ratio (GR) is defined by :

$$
G R(\%)=\frac{N-n(1)}{N}=\frac{G P}{N}
$$




\section{Case study}

\subsection{Specimen design}

Experimental studies on the tensile fatigue life of lattice metallic parts are relatively scarce. Early attempts showed the importance of having a gradient of porosity from dense parts (heads of specimens) to the middle of the structure [34] or to add fillets [35, 36], in order to avoid failure near dense parts [37] or at nodes [38]. One of the most cutting-edge designs for tensile lattice specimen was proposed by Lietaert et al. [22]. After several iterations, they achieved small scaffolds made by LBM exhibiting a linear increase of porosity from the end of the structure ( $0 \%$ porosity, fully dense) to the middle ( $80 \%$ porosity) to ensure that fatigue failure occurred in the region of interest.

In this work, the lattice structure of interest, shown in Fig. 3a, consists of a column of four octet-truss unit cells stacked on top of each other. The two unit cells in the middle can be considered as the gauge length of the fatigue specimen (see Fig. 3b). The corresponding struts have a length of $10 \mathrm{~mm}$ and a diameter of $2 \mathrm{~mm}$ (Fig. 3a). Such a diameter corresponds to previous studies performed on single struts. It allows us to rely on the S-N curve determined for example in [7]. The top and bottom unit cells are used to smooth transitions with the specimen heads. Fillets were added to avoid failure at the interface between the heads and the lattice. Conical struts in which diameter decreases from 3 to $2 \mathrm{~mm}$ were also located at the interface between bulk parts and lattice structure giving a gradient of relative density to the structure. The purpose was to localize the failure in the two central unit cells (gauge length) that are free of fillets. These choices led to a lattice structure with a bounding box $17.3 \mathrm{~mm}$ wide and $65 \mathrm{~mm}$ high.

The chosen unit cell is the octet-truss because it is a stretching-dominated lattice structure. For that reason, we can conveniently use tensile fatigue results of as-built single struts with a stress ratio of $R=0.1$. Lattice samples are manufactured vertically so that no struts are built vertically. This was motivated by our previous work [7] where it was shown that vertically produced single struts exhibit the worst tensile fatigue properties compared to tilted and horizontally produced single struts. Consequently, when the structure is loaded under tension, all struts manufactured at $45^{\circ}$ are submitted to tensile stress, while struts manufactured at $0^{\circ}$ (horizontally) are loaded in compression.

Using a column of octet-trusses has several benefits. As we aim at monitoring the failure of struts, it is convenient to be able to visually detect which strut fails and when. Note that displacement data recorded during tests confirmed their occurrence. In particular, this makes easier the experimental determination of the grace period (GP). Moreover, it also enables us to fully characterize the structure after failure with a relatively good spatial resolution, using X-ray tomography or by characterizing a given strut fracture surface. 


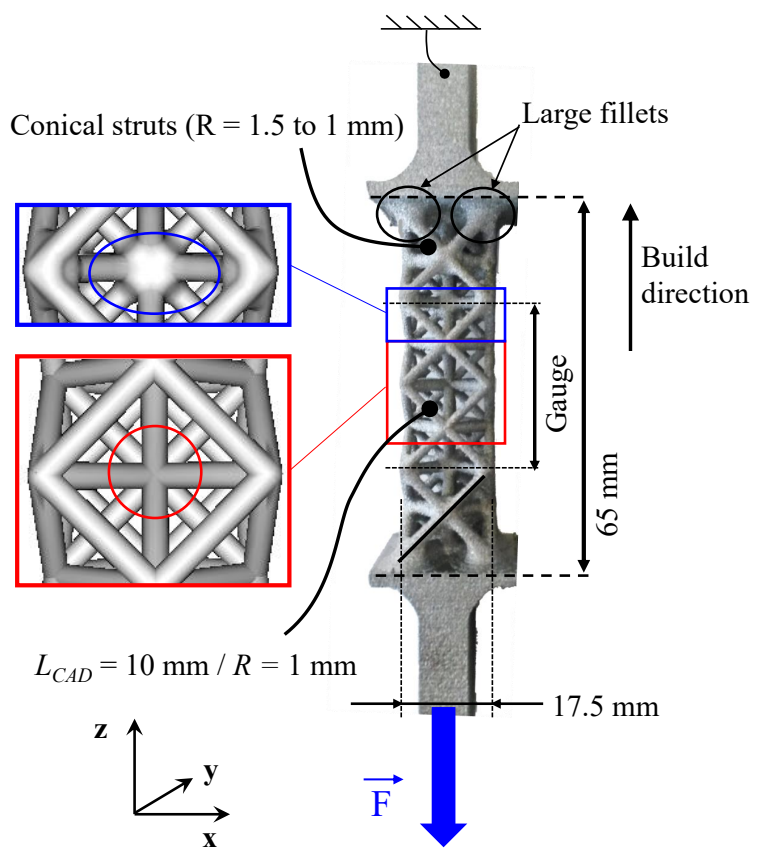

(a)

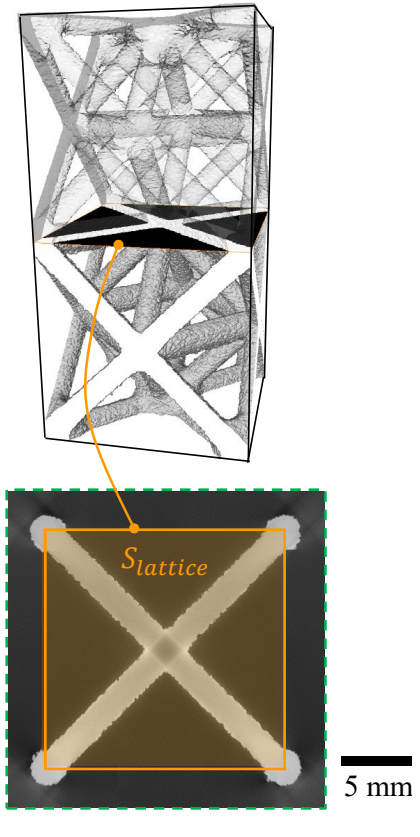

(b)

Figure 3: (a) Geometry and characteristic dimensions of the lattice specimens built by EBM and used in the present work. The specimen consists of 4 stacked octet-truss unit cells with a gradient of radius to ensure failure localization in the central gauge part. Note that there are no fillets in the gauge part. (b) Definition of the bounding box allowing to reveal the section used to compute the macroscopic stress $\left(\sigma_{\text {macro }}=F_{\text {macro }} / S_{\text {lattice }}\right)$ applied to the lattice structure based on a 3D characterization performed by X-ray microtomography. 


\subsection{Materials and experimental procedures}

\subsubsection{EBM processing conditions}

The designed lattice specimens were manufactured vertically, i.e. with their tensile axes aligned with the building direction, using an A1 EBM machine from ARCAM. A Ti-6Al-4V ELI powder whose chemical composition is given in Table 1 was used. Standard scanning strategies of ARCAM were used with a layer thickness of $50 \mu \mathrm{m}$. In this study, it is important to point out that lattice structures are fabricated with the same material on the same EBM machine and with the same scanning strategies than for single struts studied in Persenot et al. [7]. Seven lattice specimens were studied in their as-built conditions. Four additional specimens were characterized after chemical etching to evaluate the impact on the fatigue performances. To comply with [32], these specimens were dipped in a solution consisting of $48 \mathrm{~mL} \mathrm{HF} \mathrm{(48 \% ),} 160 \mathrm{~mL} \mathrm{HNO}_{3}$ and $1200 \mathrm{~mL} \mathrm{H}_{2} \mathrm{O}$ at room temperature for 30 minutes under magnetic stirring.

\subsubsection{Fatigue testing of lattice structures}

Uniaxial fatigue tests were performed in tension-tension with a constant stress amplitude $(\Delta \sigma)$, and a stress ratio $R=0.1$. Sinusoidal cyclic tests were applied at a frequency $f=5 \mathrm{~Hz}$. They were performed at room temperature using a servo-hydraulic INSTRON 8516 equipped with a $100 \mathrm{kN}$ load cell. The maximum applied stresses were smaller than half the yield stress of the lattice structure preliminary determined $\left(\sigma_{Y_{0.2 \%}} \approx\right.$ $52 \mathrm{MPa}$ ). The chosen applied stress uses the unit cell section, i.e. the square section $S_{\text {lattice }}$ bounded by the octet-truss vertices (center of nodes), and not the manufactured section (see Fig. 3b).

\subsubsection{X-ray tomography}

X-ray tomography was performed on a few samples to have a closer look at strut failure localization and to characterize in $3 \mathrm{D}$ the lattice structure. Helical scans were performed so as to characterize the lattice specimens in their full height while still having a good resolution (voxel size lower than $20 \mu \mathrm{m}$ ). Acquisition parameters were a $150 \mathrm{kV}$ acceleration voltage with a target current of $66 \mu \mathrm{A}$. Beam hardening was significant, but the use of a $0.5 \mathrm{~mm} \mathrm{Cu}$ filter and a beam hardening correction enabled contrasted volume images that could be thresholded easily using the Fiji software [39]. With this method, the relative density of the gauge of the lattice specimens is on average $16.8 \%$ in the as-built condition and $11.0 \%$ once chemically etched. The radius size of struts inside the lattice specimen can also be estimated using image analysis. Here the radius size is determined as follows: we first calculated the mean radius along every single strut having the same orientation, and then we worked out the mean and standard deviation of all these mean radii (as suggested in [7]). This was performed for the $45^{\circ}$ as-built struts and gives a radius size of $0.86 \pm 0.02 \mathrm{~mm}$, which is close to the ones obtained in the study of the fatigue behavior of single struts $(0.880 \pm 0.015 \mathrm{~mm}[7])$. This

\begin{tabular}{lcccccccc}
\hline Elements & $\mathrm{Al}$ & $\mathrm{V}$ & $\mathrm{C}$ & $\mathrm{Fe}$ & $\mathrm{O}$ & $\mathrm{N}$ & $\mathrm{H}$ & $\mathrm{Ti}$ \\
\hline wt.\% & 6.47 & 3.93 & 0.01 & 0.22 & 0.09 & 0.01 & 0.001 & Bal. \\
\hline
\end{tabular}

Table 1: Initial chemical composition of the Ti-6Al-4V ELI powder batch used in this work. 
justifies, in the numerical simulation, the use of results from Persenot et al. [7] for mean radii and standard deviations for the different strut orientations.

\subsection{Inputs of the model}

As mentioned just above, the results of [7] for radius distributions and S-N curves can be directly used as inputs for our model. The FEM software used to calculate the stress distribution inside the lattice structure is Cast3M [40] with Timoshenko beam elements (TIMO) using linear elasticity assumptions. The mesh size is $1 \mathrm{~mm}$ for struts of $11 \mathrm{~mm}$. The bottom surface nodes of the lattice structures are rigidly connected to the building and a positive force is applied on the top surface nodes.

\subsubsection{Strut properties}

Regarding the gauge part, a gaussian distribution is attributed to the beam sections to introduce the experimental scatter between all strut sections produced by EBM as determined in [7]. The mean section of struts also depends on the manufacturing orientations as shown by previous works $[33,7]$. Struts produced horizontally have a larger mean section than those produced vertically or at $45^{\circ}$. Thanks to X-ray tomography, these authors also exhibited a rather large variability of the section along a strut. Other models (e.g., [26]) slice these struts in several parts with different sections to account for this variability. Here, for the sake of simplicity and computing purposes, we assign only one radius to a given strut. The constitutive material of struts being identical, a single Young modulus and Poisson coefficient are chosen (108 GPa and 0.33 respectively [32]).

\subsubsection{Extrapolation of $S-N$ results with a Basquin law}

The numerical simulation also requires references for the S-N curves. We also take advantage of the results of Persenot et al. [7] that give the trend of the S-N curve for vertically produced single struts $\left(90^{\circ}\right)$ (Fig. 4). Each strut is assigned a specific S-N curve that is consistent with the scattering of those fatigue results. Using a Basquin law [41], experimental results (see blue line in Fig. 4) are fitted with the following equation :

$$
N \times\left(\sigma_{M A X}\right)^{k}=B
$$

with $k=4.43$ and $B=10^{15.56}$. In order to extrapolate the blue solid line in Fig. 4 to other orientations $\left(45^{\circ}\right.$ and $0^{\circ}$ struts), it is assumed that $k$ is the same for all orientations. It is also assumed that this law is valid in the area (1) $\left(N<10^{4}\right.$ cycles $)$ and $(3)\left(N>10^{6}\right.$ cycles $)$ as depicted by the green dashed line in Fig. 4 . In this work, simulated stresses are never as low as to reach $10^{6}$ cycles (area (3) in Fig. 4). Moreover, if local stresses in struts are close or larger than the yield stress (area (1) in Fig. 4), the number of cycles undergone are much too low to have an impact on the final fatigue life. Finally, the only struts loaded in tension are the $45^{\circ}$ struts, therefore they are the only struts that are likely to fail during a fatigue test. Coefficients of the Basquin law for the $\mathrm{S}-\mathrm{N}$ curve of the $45^{\circ}$ struts are $k=4.43$ and $B=10^{16.1}$. 


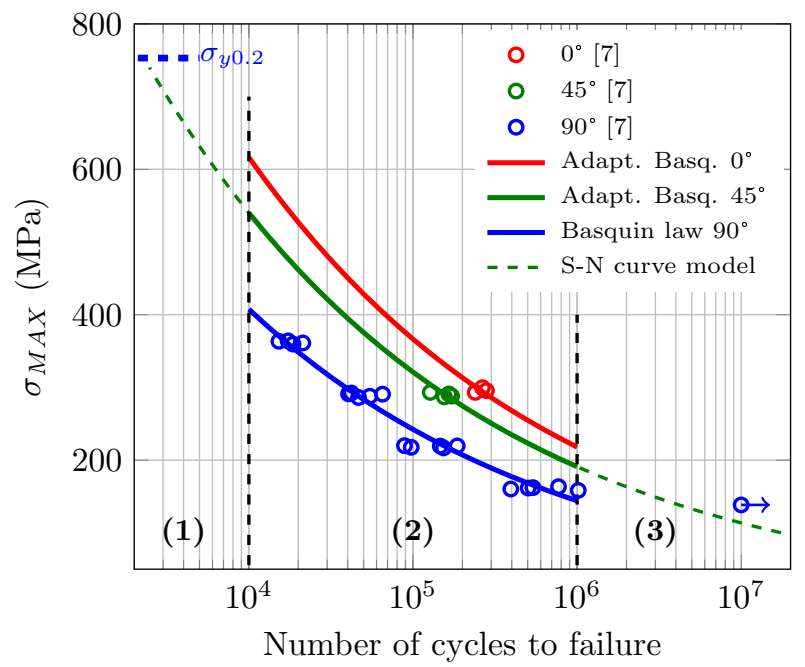

Figure 4: S-N curves of as-built single struts (adapted from [7]). Circles stand for experimental fatigue results for three orientations $0^{\circ}, 45^{\circ}$ and $90^{\circ}$ with respect to the building direction. The blue solid line is a fit following Basquin law [41] on the vertical single strut results $\left(90^{\circ}\right)$, while other solid lines show the extrapolation of the Basquin law for other orientations. Dashed black lines divide the diagram into three areas. Area (1) corresponds to the low cycle fatigue regime, meaning the Basquin law is hardly valid in this region as important plastic strain may be involved. Area (2) limits the proper use of the Basquin law in the region $10^{4}-10^{6}$ cycles. Area (3) is associated with the endurance limit, therefore a stress plateau is usually achieved.

\subsection{Fatigue life and time period of grace predictions}

\subsubsection{As-built lattice structures}

Results of experimental fatigue tests are shown as red squares in Fig. 5. Hollow squares represent the number of cycles of the first strut that failed, as detected during experiments. Solid squares depict the fatigue life of the lattice. Seven as-built samples were tested (three, two and two samples at $25 \mathrm{MPa}, 21 \mathrm{MPa}$ and $17 \mathrm{MPa}$ respectively). The failure of the lattice is not instantaneous: struts fail one after the others, with a certain delay between each event. The delay between the first strut failure event and the total failure of the lattice can be viewed as a grace period, during which the structure progressively fails but still sustains the macroscopic stress. It is interesting to note that, for all samples, the grace period has a mean value ranging in the interval [one fifth - one quarter] of the fatigue life, whatever the stress level.

To predict the fatigue life, the grace period and the grace ratio of the lattice specimens, a first batch of simulations was run. Results are shown in Fig. 6 for one level of stress (17 MPa). 3D X-ray characterization on single struts [7] have shown that the radius size is normally distributed around $0.88 \mathrm{~mm}$ for $45^{\circ}$ struts and around $0.92 \mathrm{~mm}$ for $0^{\circ}$ struts as illustrated in Fig. $6 \mathrm{~b}$ (with standard deviations of $0.015 \mathrm{~mm}$ ). To account for this variability, five samples have been drawn taking into account this distribution (Fig. 6a-b). The effect of the strut radius distribution is first studied in the case of a unique S-N curve (from Fig. 6c to e), i.e. all struts have exactly the same fatigue behavior. The mean number of cycles to failure is $86 \cdot 10^{3}$, while minimum and maximum values are $82 \cdot 10^{3}$ and $92 \cdot 10^{3}$ cycles respectively. Similarly, extreme values for these five samples give a grace ratio of $23.7 \%$ (minimum) and $33.7 \%$ (maximum) meaning that there is a significant scatter. The mean value is also reported in Fig. $6 \mathrm{c}: 28.2 \%$ which is close to the experimental range of values. 


\begin{tabular}{|c|c|c|c|}
\cline { 2 - 4 } \multicolumn{1}{c|}{} & $\begin{array}{c}\text { Nb of cycles } \\
\text { to failure }\left(\mathrm{x} 10^{3}\right)\end{array}$ & $\begin{array}{c}\text { Grace Period } \\
(N-n(1))\left(\mathrm{x} 10^{3}\right)\end{array}$ & $\begin{array}{c}\text { Grace Ratio } \\
\left(\frac{N-n(1)}{N}\right)(\%)\end{array}$ \\
\hline $25 \mathrm{MPa}$ & $16.6 / 19.3 / 16.3$ & $4.1 / 5.3 / 4.8$ & $24.6 / 27.3 / 29.6$ \\
$21 \mathrm{MPa}$ & $31.5 / 26.0$ & $6.9 / 6.5$ & $21.8 / 24.9$ \\
$17 \mathrm{Mpa}$ & $97.8 / 93.0$ & $17.9 / 22.6$ & $18.3 / 24.3$ \\
\hline
\end{tabular}

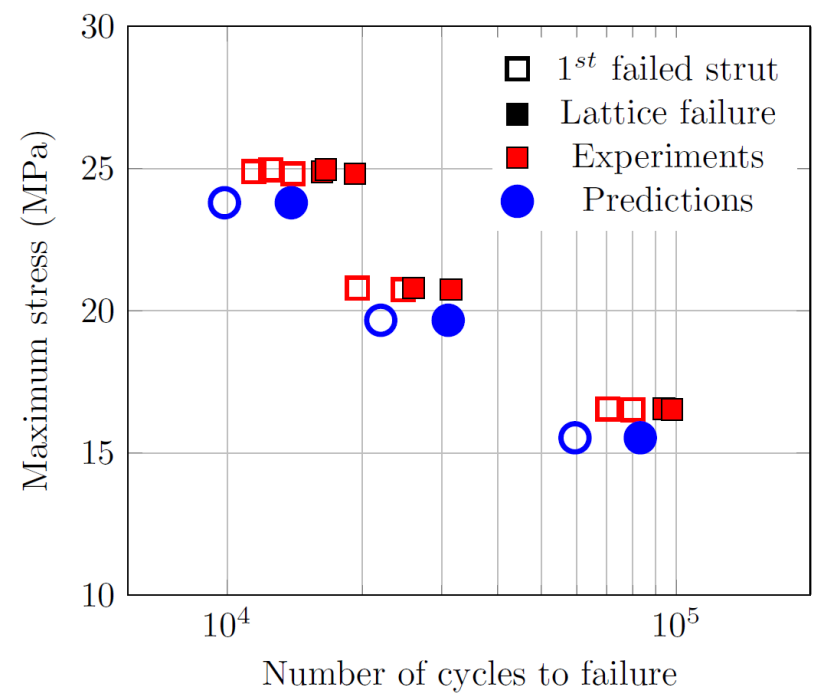

Figure 5: Table summarizing experimental fatigue results for as-built lattice specimens and S-N results. Red squares stand for experimental results and blue points stand for predictions (with a $\sigma$ offset of $-1 \mathrm{MPa}$ for the sake of clarity). Hollow symbols are associated with the failure of the first strut within the lattice while filled symbols correspond to the final failure of the lattice specimen. This leads to 100 full simulations (from start to failure) for each of the three stress levels investigated. Errors calculated based on the standard deviation of simulation results are not significant enough to be reported $(\approx 4 \%)$ on this figure. Errors were estimated using 5 draws of radius distribution and for every radius size distribution, 20 draws of the single strut S-N curves. 
(a)

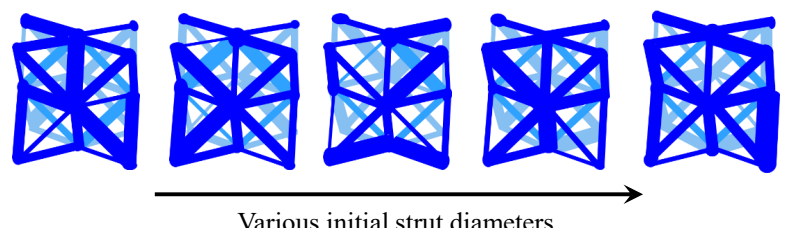

Various initial strut diameters

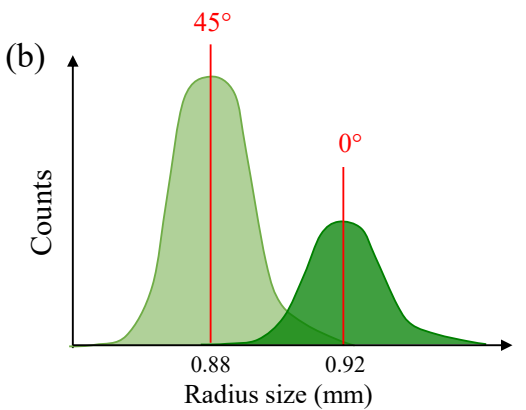

(d)
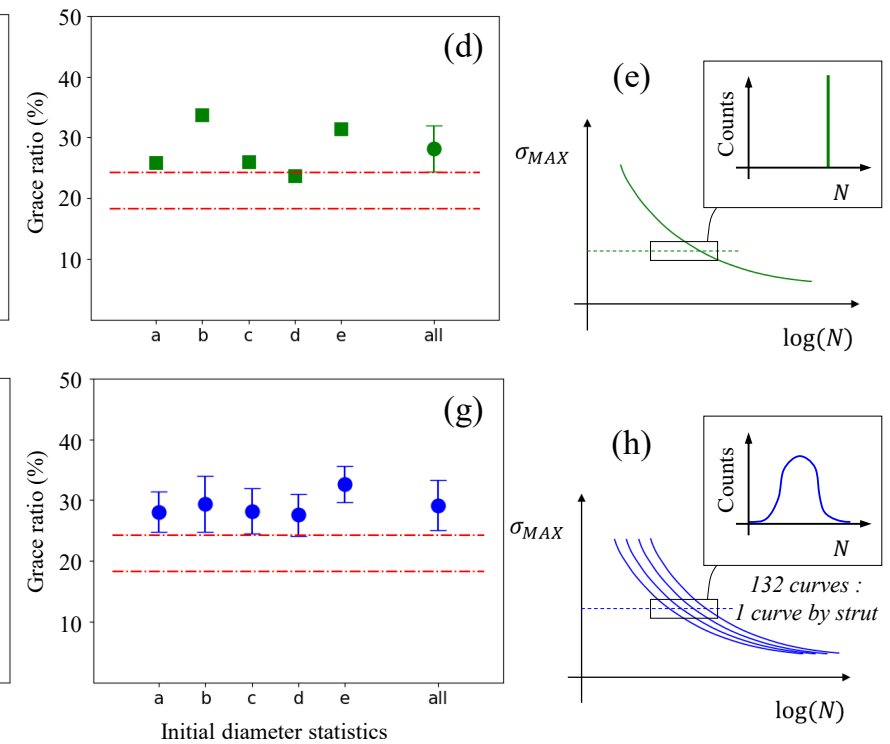

Initial diameter statistics

(h)

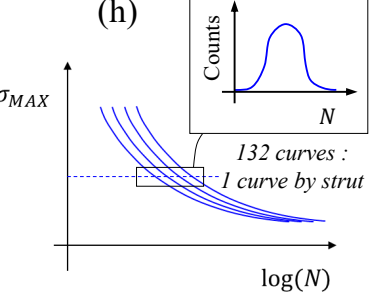

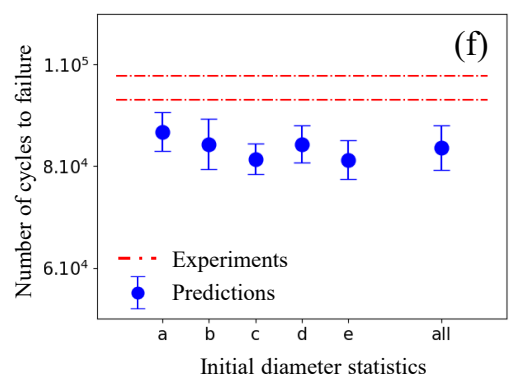

Figure 6: Sensitivity analysis on the fatigue life $(c, f)$ and on the grace ratio $(d, g)$ for various initial strut diameters and S-N curves at macroscopic stress of $17 \mathrm{MPa}$. (a) Schematic giving a few examples of octet-truss unit cells with varying initial strut diameters that exhibit a radius size distribution illustrated in (b). $45^{\circ}$ struts have a mean diameter of $0.88 \mathrm{~mm}$ and $0^{\circ}$ struts (horizontal) have a mean diameter of $0.92 \mathrm{~mm}( \pm 0.015 \mathrm{~mm})$. Green squares on (c) and (d) give results in the case of a unique S-N curve, i.e. with no scattering as shown in Fig. 4 and sketched in (e). All simulations reported in (f-g) use a different draw of the S-N curve distribution (typical histogram of S-N curves is sketched in (h). 5 draws are performed for the strut radii, and in those draws, 20 additional random draws of the S-N curves are made. Red dash-dot lines illustrate the results of the two experimental specimens tested at $17 \mathrm{MPa}$. 
In a second step, the sensitivity of fatigue life and grace ratio to scatter in the S-N curves has been investigated. Results are shown in Fig. 6f-g. For a given draw of a lattice structure, each of the 132 struts of the lattice is randomly assigned an S-N curve. For each one of the five lattice samples previously created, 20 different samplings of S-N curves were generated using the same normal distribution as illustrated in Fig. $6 \mathrm{~h}$. A random sampling of $5 \times 20=100$ different initial configurations is then achieved. These inputs (distribution of S-N curves and strut radii) are chosen to agree with the as-built single strut study. Note that the corrective factor $\lambda$ introduced in Eq. 10 was fixed to a unique value $\lambda=1.47$ in this section.

In this case of multiple S-N curves (Fig. 6h), results are reported using average values and standard deviations as error bars in Fig. 6f-g. Using one distribution of S-N curves seems to lower the variability for the fatigue life as well as for the grace ratio. The difference between the minimum and maximum of the average values reaches $5 \cdot 10^{3}$ for the fatigue life and about $5 \%$ for the grace ratio. Average values are relatively close to the case of a unique S-N curve $\left(83.6 \cdot 10^{3} \pm 4.4 \cdot 10^{3}\right.$ for the fatigue life and $29.1 \% \pm 4.1 \%$ for the grace ratio).

Interestingly, using only one unique $\mathrm{S}-\mathrm{N}$ curve and different draws of the radius distribution gives results similar to those obtained with scattered S-N curves, at least for the particular distributions of strut radii and $\mathrm{S}-\mathrm{N}$ curves investigated here. In other words, radius distribution seems to account as a first-order parameter, whereas S-N curve distribution accounts as a second-order one for both the prediction of grace ratio as well as the fatigue life.

Numerical simulations were performed at different stress levels and the results are shown as blue circles and dots in Fig. 5 with standard deviations represented by the size of dots. The comparison with experimental results is risky considering the few numbers of tested specimens. Broadly speaking, fatigue life is underestimated for all stress levels. This underestimation must be related to the uncertainty in the local stress values, i.e. to the coefficient $\lambda$. This point will be further discussed in section 4 . The grace ratio, in turn, is slightly over-estimated by simulations (mean value of $28.4 \% \pm 3.9 \%$ ) as already observed in Fig. $6 \mathrm{~d}$ and g, and seems to be independent of the macroscopic stress.

\subsubsection{Extending the variability}

In section 3.4.1, we have only investigated the effect of several draws of a given radius size distribution (see Fig. 6b), and the effect of several draws of S-N curves with a given variability (see Fig. 6h). These inputs were chosen to match the experimental data obtained from as-built single struts. One of the main advantages of performing numerical simulations is the possibility to explore deeper the configuration space. Depending on the scanning strategies, the radius distribution could be more scattered, or surface defects more heterogeneously spread. In this section, we aim at studying the effect of standard deviations for both the size and the S-N behavior of single struts. Fig. 7 illustrates the sensitivity of the numerical predictions to four different cases. Two different radius size distributions and two different $\mathrm{S}-\mathrm{N}$ curve distributions are investigated. In both cases, the mean values are the same. Only the standard deviation is varied.

The first case is the one used in section 3.4.1, with distributions of radii and of S-N curves that are the 


\begin{tabular}{c|c|c|c|c} 
Strut orientation & \multicolumn{2}{|c|}{$0^{\circ}$} & \multicolumn{2}{c}{$45^{\circ}$} \\
\hline Distribution & $R_{m}$ & std & $R_{m}$ & std \\
\hline A & 0.92 & 0.015 & 0.88 & 0.015 \\
B & 0.92 & 0.05 & 0.88 & 0.05
\end{tabular}

Table 2: Summary of strut radius distribution used. $R_{m}$ is the mean radius and std the standard deviation to the mean.

\begin{tabular}{c|c|c|c} 
Distribution & $1^{\text {st }}$ peak weight & $2^{\text {nd }}$ peak weight & standard deviation \\
\hline SN-1 & - & - & $\delta$ \\
SN-2 & - & - & $2 \delta$ \\
SN-3 & $2 / 3$ & $1 / 3$ & $\delta$ \\
SN-4 & $1 / 3$ & $2 / 3$ & $\delta$
\end{tabular}

Table 3: Summary of the S-N curves characteristics used in this work. In the case of bi-modal distributions, columns 1 and 2 denote the proportion of S-N curves centered around the two peaks.

closest to the as-built single strut study. The corresponding distributions are denoted A (for the radius) and $\mathrm{SN}-1$ (for the fatigue curve). A second radius distribution (denoted B in Fig. 7a) is proposed to keep the same mean values for $45^{\circ}$ struts and $0^{\circ}$ struts but a larger standard deviation of $0.05 \mathrm{~mm}$ instead of $0.015 \mathrm{~mm}$ for the distribution A. Similarly, the sampling distribution of S-N curves for the distribution SN-2 exhibits the same mean value as $\mathrm{SN}-1$, but the standard deviation is doubled (see schematic in Fig. 7b). This leads to four different configurations: A/SN-1, A/SN-2, B/SN-1, and B/SN-2. For each radius distribution, five draws are performed, and for every one of them, 20 samples of S-N curves are generated.

Results are shown in Fig. 7c,d and e for the fatigue life, the grace period and the grace ratio respectively. There is clear evidence of a strut size distribution effect for $N$ and $G R$. The fatigue life significantly decreases when the spread of radius distribution is larger, the mean value dropping from $\approx 82 \cdot 10^{3}(\mathrm{~A} / \mathrm{SN}-1)$ to $\approx 65 \cdot 10^{3}$ (B/SN-1). The effect of the spread of distribution for S-N curves is much less pronounced. As already noticed in the previous section, it seems, at least in the range of standard deviations studied here (see Table 3), that the effect of radius size distribution is of the first order. The grace period being roughly the same for all combinations (see A/SN-1, A/SN-2, B/SN-1, B/SN-2 in Fig. 7d), an early failure of the first strut can lead to an early failure of the lattice structure (as observed for distribution B in Fig. 7c). In fact, a scattered radius distribution suggests a larger stress contrast from one strut to another in the structure, leading to early failure of the first strut. Consequently, the grace ratio increases for distribution B on average (Fig. 7e) with the mean value increasing from $\approx 30 \%$ to nearly $45 \%$. 
(a) Radius distribution
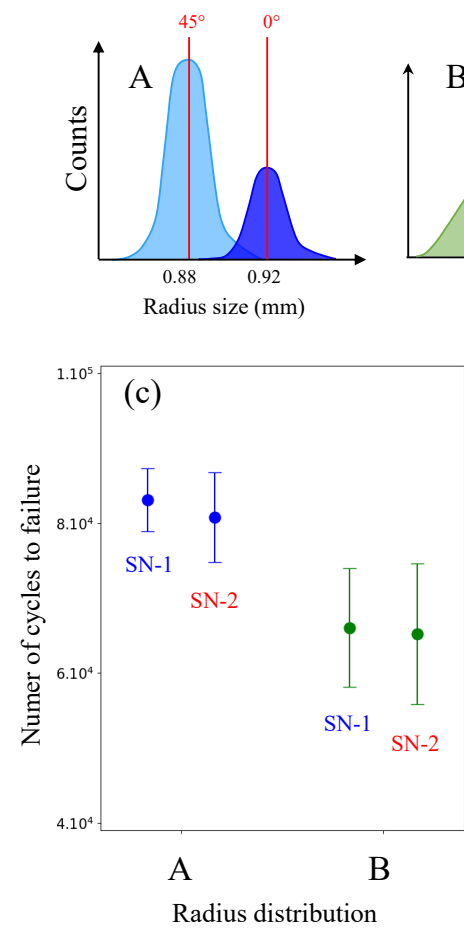

(b) Batches of S-N curves
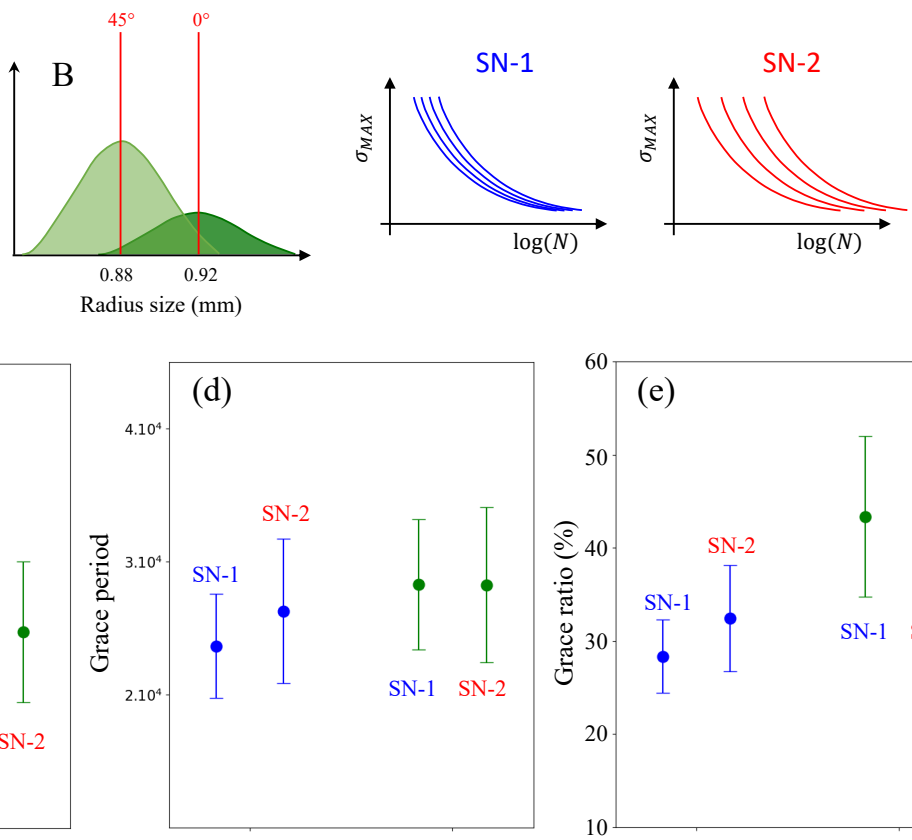

A

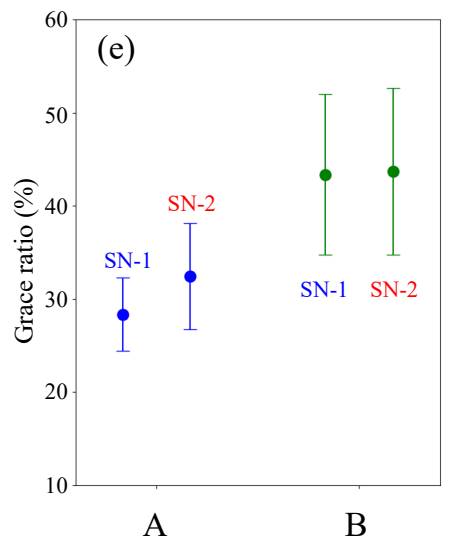

A

B

Figure 7: Sensitivity analysis of the model with (a) different radius size distribution and (b) different samples of S-N curves (both distributions are centered around 157,000 cycles at $290 \mathrm{MPa}$ ). 5 draws of distributions A and B are used. For each of these draws, 20 samples of $132 \mathrm{~S}-\mathrm{N}$ curves are used for both distributions SN-1 and SN-2. (c) Fatigue life, (d) grace period and (e) grace ratio predictions for an applied load of $4 \mathrm{kN}$. 100 simulations are performed to compute the standard deviations and provide error bars. 
(a) Radius distribution

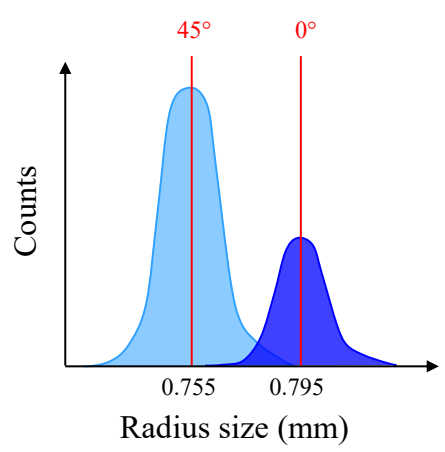

(b) Batches of S-N curves

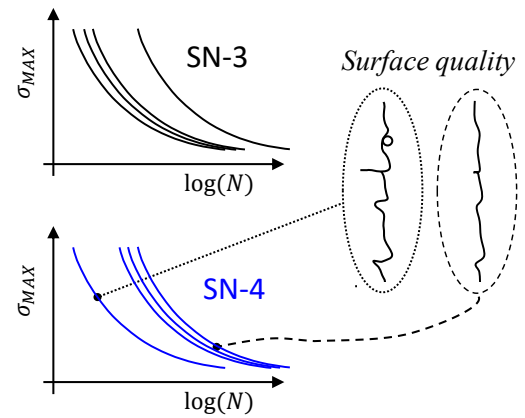

\begin{tabular}{|c|c|c|c|c|c|c|}
\cline { 2 - 7 } \multicolumn{1}{c|}{ (c) } & $\begin{array}{c}\text { Mean of nb of cycles } \\
\text { to failure }\left(\mathrm{x} 10^{3}\right)\end{array}$ & Std $\left(\mathrm{x} 10^{3}\right)$ & $\begin{array}{c}\text { Mean of grace } \\
\text { period }\left(\mathrm{x} 10^{3}\right)\end{array}$ & Std (x10 $)$ & Grace Ratio (\%) & Std (\%) \\
\hline SN-3 & 105.3 & 8.0 & 33.3 & 7.7 & 31.4 & 5.7 \\
SN-4 & 125.4 & 14.4 & 46.9 & 10.8 & 37.2 & 6.4 \\
\hline
\end{tabular}

Figure 8: Sensitivity analysis of the model for a given radius size distribution (a), and (b) different samples of S-N curves using two bi-modal distributions (SN-3 and SN-4) shown at 360 MPa. Peaks of distribution SN-3, and 4 are centered around 113,000 and 215,000 cycles at $360 \mathrm{MPa}$. Distribution characteristics are given in Table 3. (c) Predictions results for the number of cycles to failure, grace period and grace ratio (mean and standard deviations are given).

\subsubsection{Applications to etched lattice structures}

The normal distributions used previously can be seen as an ideal and common case. More complex distributions may arise, such as in the case of etched lattices presented in this section. Previous studies on etched single struts $[42,32]$ have shown some interesting results: the surface quality is improved through chemical etching but strongly depends on the etching time. For short etching time, a substantial number of critical defects remain, leading to fatigue lives similar to those obtained for as-built specimens. Some rare single struts exhibit larger fatigue life. On the contrary, for longer etching time, the majority of the struts exhibit good fatigue life properties. Only a few of them still present severe critical defects. These two situations can be respectively represented, in terms of S-N curves by the two bi-modal distributions SN-3 and $\mathrm{SN}-4$, as shown in Fig. 8. For the numerical application, only one distribution of radius size mimicking the one determined on etched single struts [43] has been used (again with five different draws). Coefficients of the Basquin's law are modified accordingly for etched single struts $\left(k=4.43\right.$ and $\left.B=10^{16.6}\right)$. As previously mentioned, the stress corrective coefficient $\lambda$ is fixed at 1.62 in this case. The density of etched lattice specimen being about 35\% smaller than as-built ones, a reduced macroscopic stress of $12 \mathrm{MPa}$ is applied to target a fatigue life of approximately $10^{5}$ cycles. The bi-modal distributions SN-3 and SN-4 are centered around 113,000 cycles for the first peak and around 215,000 cycles for the second peak (see Table 3 for a summary of all the S-N curve distribution characteristics).

The poor results of SN-3 are straightforward. Failed struts had most likely an S-N curve contained in 
the first peak of the distribution. SN-4 distribution markedly gives larger fatigue life, grace period and grace ratio than the $\mathrm{SN}-3$ distribution. In this case, the increase of grace period likely comes from the early failure of a few struts with poor fatigue properties, and the likelihood to have most of the struts with good fatigue properties that will fail afterward. Indeed, the mean value of $n(1)$ is close between SN-3 and SN-4 $\left(72 \cdot 10^{3}\right.$ and $78.5 \cdot 10^{3}$ respectively), while the mean fatigue life of SN-4 is higher $\left(20 \cdot 10^{3}\right.$ more cycles) than for SN-3.

From an experimental point of view, a few lattice specimens were post-treated using chemical etching in order to decrease the harmfulness of surface notch-like defects. Interestingly, the fatigue tests reported in Fig. 9 for etched specimens gave a larger grace ratio in comparison with as-built lattice specimens independently of the stress. A specific property of the lattice structure (stress normalized by the density) is exhibited in Fig. 5c. In this scheme of presentation, the benefit of etching a lattice structure is clearly evidenced in terms of $N, G P$, and $G R$.

As already observed in section 3.4.2, results on etched single struts [43] were scattered, with some struts failing early during experiments. This means that despite a homogeneous etching, a few severe residual notch-like defects may remain in some struts. In a lattice structure, this translates to an increase of the grace period.

Finally, the etching time used to post-treat our lattice structures seems to be correctly described by the SN-4 distribution: the number of cycles to failure is well predicted $\left(125 \cdot 10^{3} \pm 14 \cdot 10^{3}\right.$ cycles at $\left.12 \mathrm{MPa}\right)$ as well as the grace period and grace ratio (respectively $47 \cdot 10^{3} \pm 11 \cdot 10^{3}$ cycles and $37 \% \pm 7 \%$ ). 


\begin{tabular}{|c|c|c|c|}
\cline { 2 - 4 } \multicolumn{1}{c|}{ (a) } & $\begin{array}{c}\text { Nb of cycles } \\
\text { to failure }\left(\mathrm{x} 10^{3}\right)\end{array}$ & $\begin{array}{c}\text { Grace Period } \\
(N-n(1))\left(\mathrm{x} 10^{3}\right)\end{array}$ & $\begin{array}{c}\text { Grace Ratio } \\
\left(\frac{N-n(1)}{N}\right)(\%)\end{array}$ \\
\hline $19 \mathrm{MPa}$ & $18.6 / 20.9$ & $5.4 / 8.3$ & $29.1 / 39.4$ \\
$12 \mathrm{Mpa}$ & $129.6 / 141.4$ & $54.6 / 43.4$ & $42.1 / 30.7$ \\
\hline
\end{tabular}

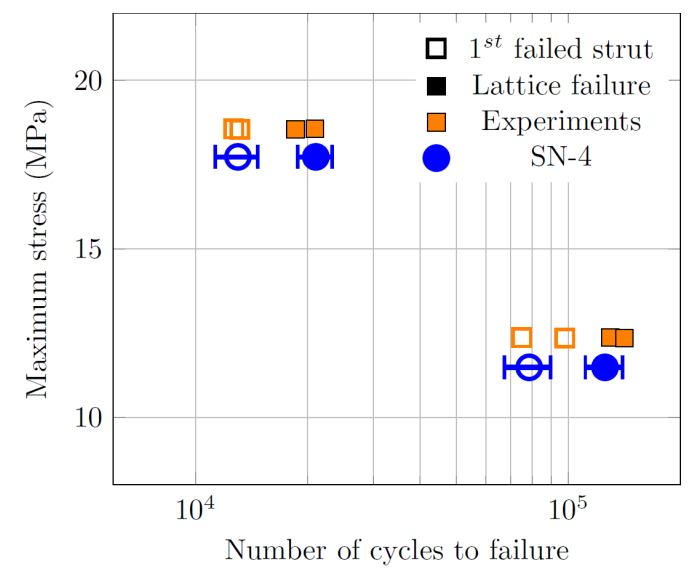

(b)

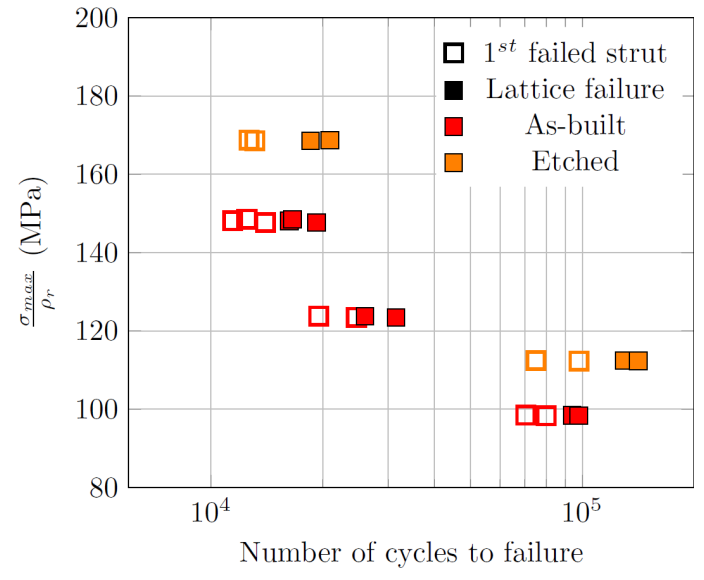

(c)

Figure 9: (a) Table summarizing experimental fatigue results for etched lattice specimens. (b) S-N results: Orange squares stand for experimental results in the case of etched lattice structures. Hollow symbols are used for the failure of the first strut. Blue points give the mean results for the SN-4 predictions (with a $\sigma$ offset of $-1 \mathrm{MPa}$ for the sake of clarity). Error bars give the standard deviation obtained using 5 different initial states ( 5 draws of radius distribution) and 20 samples of S-N curves for the single struts, meaning 100 different simulations for each stress level (see Fig. 8 for details). (c) Maximum stress normalized by the density as a function of the fatigue life for as-built (red) and etched (orange) lattice structures. 


\section{Discussion}

\subsection{Chronology of the failure events}

A further step consists in focusing on the chronology of failed struts. This was achieved experimentally. As already detailed in section 3.1, lattice structures were specifically designed to be able to visually detect the failure of struts. As tests are performed at constant macroscopic stress, the displacement data confirmed the occurrence of a failure and gave the corresponding number of cycles during the test. Complete failure scenarios were monitored. In other words, the successive failure of every strut was recorded during the tests.

This section aims to assess the ability of the presented model to predict correctly such a chronology. To that end, beam element simulations were performed on configurations observed experimentally: at each step, the beam corresponding to the observed broken strut was removed from the numerical lattice: the order observed experimentally was then reproduced numerically. Four different experimental scenarios have been reproduced. Some steps of the simulation performed on one of the experimental samples are displayed in Fig. 10a. The failure of struts during this experiment leads to a redistribution of stresses which is evidenced (see the color bar). For the sake of clarity, no radius distributions were used in the simulations. Radii of $45^{\circ}$ struts were set to $0.88 \mathrm{~mm}$ and radii of $0^{\circ}$ struts to $0.92 \mathrm{~mm}$.

At each step of the simulation, let us denote $\sigma_{\text {strut }}$ the stress value of the strut which actually failed experimentally and $\sigma_{m}$ the maximum stress among the remaining struts of the lattice. $\sigma_{m}$ is recalculated every step. If the numerically most loaded strut has experimentally failed, the ratio $\frac{\sigma_{s t r u t}}{\sigma_{m}}=1$.

In Fig. 10b, this ratio is represented as a function of the number of failed struts in lattice structures. The different numerical results of experimental scenarios are depicted: two as-built and two etched lattice specimens.

It appears that the strut that fails is rarely the one sustaining the maximum stress, but is usually not far

away from this maximum. From time to time, its stress level is very low (below $\frac{1}{2} \sigma_{m}$ ). It means that stress alone can not be used to predict the strut that fails. This supports the idea to use a damage accumulation law. In the present paper, we focused on classical Miner's rule. Its use can be questionable. Several other damage laws exist as pointed out by Hwang and Han [44]. However, we relied on Miner's rule broad use in the fatigue community and took advantage of its relative simplicity.

For statistical purposes, a hundred simulations were performed, using the damage accumulation law with both the SN-1 and SN-4 distributions (see Table 3), for as-built and etched lattices respectively. Corresponding values are reported in Fig. 10b. For instance, simulations predict for as-built lattices that roughly $60 \%$ of failed struts have broken in the range $\sigma_{\text {strut }}=80-100 \%$ of $\sigma_{m}$. This value rises to about $79 \%$ in the case of etched lattices. It seems consistent with the fact that the fatigue behavior of etched struts is less sensitive to critical defects, and more driven by the stress value.

In general, trends are correctly captured, with a good ability to predict the failure of struts, though they do not sustain the maximum stress. This proves the efficiency of the damage accumulation model to predict tendencies and global values, even if local events are not fully described. 


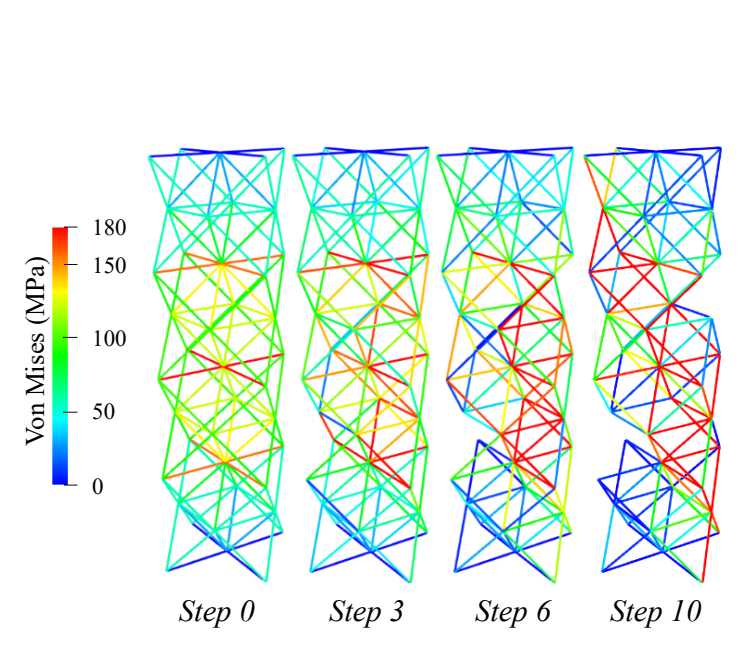

(a)

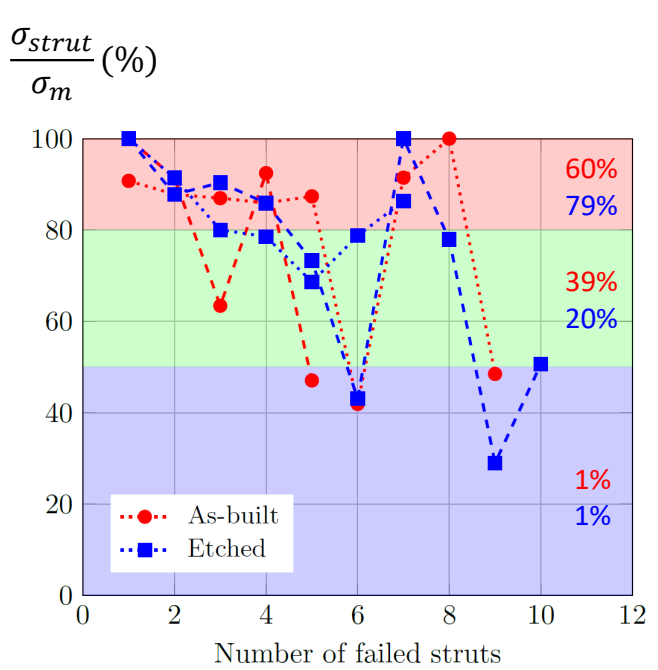

(b)

Figure 10: (a) Finite element simulation using Timoshenko beam elements of a given experimental scenario (applied macroscopic stress $\sigma=12 \mathrm{MPa}$ ). As struts fail, stresses are redistributed. (b) Ratio of the normal stress calculated in struts $\left(\sigma_{\text {strut }}\right)$ just before they fail over the maximum tensile stress within the lattice structure $\left(\sigma_{m}\right)$, for four different experimental scenarios $(2$ as-built and 2 etched specimens). $\sigma_{m}$ is re-calculated every step. Simulations on 1200 struts predict for as-built lattices that failed struts are $\approx 60 \%$ of the time in the range $80-100 \% \times \sigma_{m}, \approx 39 \%$ of time in the range $50-80 \% \times \sigma_{m}$ and almost never below $\frac{1}{2} \sigma_{m}$ (respectively $\approx 79 \%, \approx 20 \%$ and $\approx 1 \%$ for etched lattices). 


\subsection{Considerations on stress estimation at the strut level}

In the present model, a stress level per strut is defined. This value is chosen to be able to use the experimental data contained in the S-N curves and thus to predict the failure of the corresponding strut. It is interesting to locate the crack that leads to failure in a strut. Using X-ray tomography, Fig. 11a illustrates in blue color the cracks observed in the lattice structure after a fatigue test. It is worth noting that all cracks were located close to nodes in all our fatigue experiments. In the work of Latture et al. [38], the compression behavior of octet-truss structures with and without fillets was compared. They observed that failure occurs at nodes for the octet-truss without fillets and near nodes for filleted ones. Our observations in tensile loading are consistent with the ones reported by Latture et al. [38]. However, under cyclic loading, failure occurs close to but not at nodes (Fig. 11a). The full 3D finite element simulation proposed in Fig. 11b shows large stress concentrated areas around nodes. In fact, strut failure results from combined effects of stress concentration and surface notch-like defects, as recently discussed by Persenot et al. [7] or Romano et al. $[45]$.

X-ray microtomography showed that the nodes did not contain more or largest defects than anywhere else in the structure. Only their presence and harmfulness in regions submitted to larger stresses makes them the main initiators of fatigue cracks resulting in crack localization near nodes and not at nodes. Different sources of stress concentration near nodes exist: geometrical singularities (e.g. absence of fillets) or bending stresses. In particular, the second ones are present when nodes are rigid or when the lattice is no more a stretching dominated one (in the present case, as soon as the first strut has failed). Portela et al. [46] have already discussed the ambiguity of the bending and stretching dominated behavior classification commonly reported in the literature [47], as they emphasize the importance of correctly modeling nodes. In any case, from a numerical point of view, a full 3D finite element simulation should be required for a correct estimation of the local stress levels.

In the present work, we assumed a stretching-dominated behavior, allowing us to rely on the definition of stress given in Eq. 10. Moreover, we introduced the corrective coefficient $\lambda$ that integrates into a single value the contributions to the stress of bending moments, rigid nodes and geometrical singularities. Despite this strong assumption and the lack of local description of stress, predictive results are in relatively good agreement with experiments, and fast computing is possible and enables us to perform statistical analyses. 


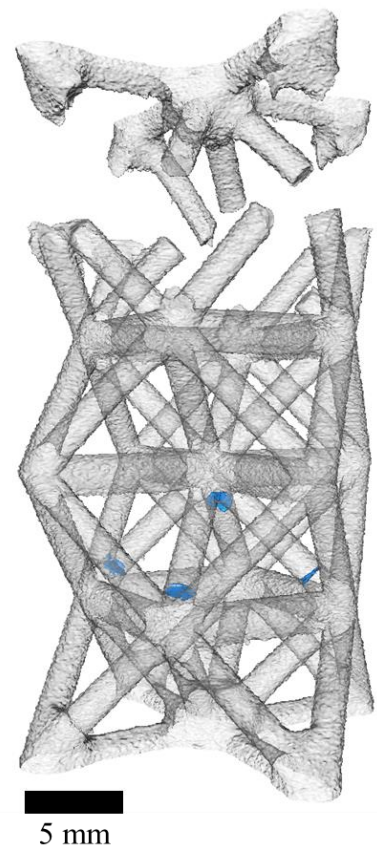

(a)

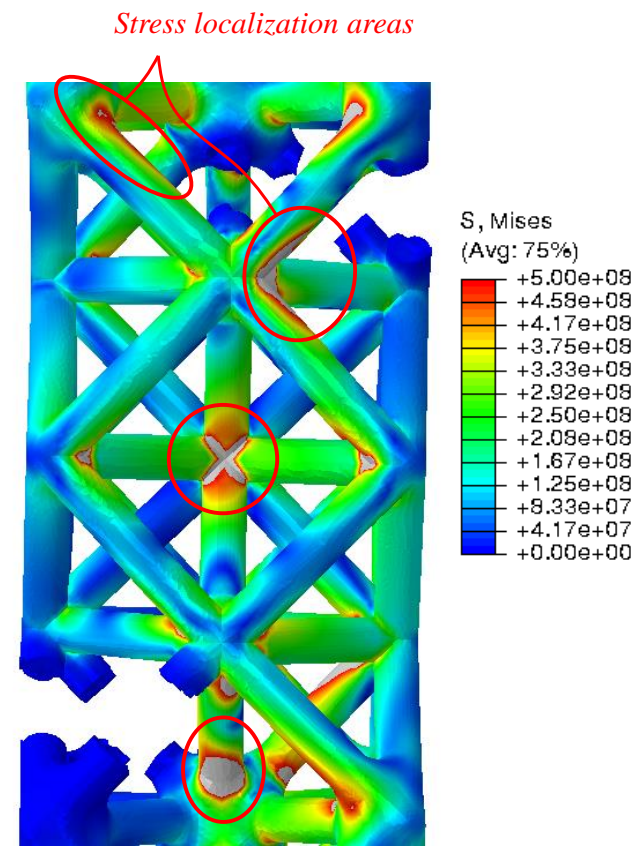

(b)

Figure 11: (a) 3D tomographic reconstruction of an as-built specimen after failure. Blue areas show strut failure locations as-revealed by X-ray tomography. In a sake of clarity, note these cracks were dilated. (b) Full volume finite element calculation results of the Von Mises equivalent stress when considering that 10 struts had already failed under a tension load of $3 \mathrm{kN}$ (same scenario than in Fig. 10a). Simulations are performed with ABAQUS/CAE Standard Explicit [48] with a linear elastic behavior of the material. 


\section{Conclusions}

The main conclusions of the present work can be drawn as follows:

- From an experimental point of view, fatigue failure of lattice specimens was shown to be gradual, leading to substantial stress redistribution.

- A numerical framework based on a cascading failure model to predict the fatigue life of lattice structures has been proposed.

- Damage within the lattice is estimated thanks to the Miner's rule.

- The grace ratio, a characteristic of the progressive failure of a lattice, was introduced. It is defined as a percentage of fatigue life during which the structure holds in spite of being damaged.

- Properties of individual struts constituting the lattice can be chosen independently in our model. The effect of two strut parameters on the fatigue properties of lattice specimens, namely the spread of the radius distribution in the lattice, and the S-N curve distribution of single struts have been studied. An increase of the spread of the radius size distribution, or an increase of the dispersion of the S-N curve distribution, led to an increase of the grace ratio and a decrease of the fatigue life.

- Predictions of the fatigue life, grace period and grace ratio using our model are in relatively good agreement with experimental results when using inputs, i.e. radius distribution and S-N curve distribution determined experimentally on single struts.

- A few limits are still hindering the accuracy of our predictions, in particular stress concentrations near nodes, a possible bending contribution to the stress, and radius variability along a given strut. To overcome those limits, a corrective coefficient was introduced to estimate stresses in struts. A finer determination of this corrective coefficient should require full finite volume element calculations all along the cascading failure. Such a systematic procedure was out of the scope of this study.

The main conclusion is that there is an interplay between the desired fatigue properties and the chosen strut parameters (radius, stiffness) to design lattice structures. Moreover, it could be interesting to determine if the grace ratio is topologically driven. Thus, such a numerical tool can be considered as a first step towards optimization of the lattice structure fatigue properties.

\section{Competing interests}

The authors declare no financial or personal conflict of interests that could have influenced the present work. 


\section{Acknowledgements}

The authors would like to thank the French "Agence Nationale de la Recherche (ANR)" and the French "Fondation de Recherche pour l'Aéronautique et l'Espace (FRAE)" for the financial support of this project entitled "Fabrication Additive et FAtigue de Structures Cellulaires Intégrées en AEronautique" (FA2SCINAEANR-15-CE08-0038-03). Partners from Airbus (Philippe Emile and Claude Archambeau) are also acknowledged for fruitful collaboration. This work was performed within the framework of the Center of Excellence of Multifunctional Architectured Materials "CEMAM" nAN-10-LABX-44-01 funded by the "Investments for the Future Program".

\section{Data availability}

The raw and processed data required to reproduce these findings cannot be shared at this time as the data also forms part of an ongoing study.

\section{References}

[1] N. Fleck, V. Deshpande, M. Ashby, Micro-architectured materials: past, present and future, Proceedings of the Royal Society A: Mathematical, Physical and Engineering Sciences 466 (2121) (2010) 2495-2516.

[2] M. Ashby, Designing architectured materials, Scripta Materialia 68 (1) (2013) 4-7.

[3] Y. Brechet, J. D. Embury, Architectured materials: Expanding materials space, Scripta Materialia 68 (1) (2013) 1-3.

[4] P. Edwards, A. O'Conner, M. Ramulu, Electron Beam Additive Manufacturing of Titanium Components: Properties and Performance, Journal of Manufacturing Science and Engineering 135 (6) (2013) 061016. doi:10.1115/1.4025773.

[5] M. Kahlin, H. Ansell, J. J. Moverare, Fatigue behaviour of notched additive manufactured Ti6Al4V with as-built surfaces, International Journal of Fatigue 101 (2017) 51-60. doi:10.1016/j.ijfatigue.2017. 04.009.

[6] D. Greitemeier, F. Palm, F. Syassen, T. Melz, Fatigue performance of additive manufactured TiAl6V4 using electron and laser beam melting, International Journal of Fatigue 94 (2017) 211-217. doi:10. 1016/j.ijfatigue.2016.05.001.

[7] T. Persenot, A. Burr, G. Martin, J.-Y. Buffiere, R. Dendievel, E. Maire, Effect of build orientation on the fatigue properties of as-built electron beam melted ti-6al-4v alloy, International Journal of Fatigue 118 (2019) 65-76.

[8] V. Chastand, P. Quaegebeur, W. Maia, E. Charkaluk, Comparative study of fatigue properties of Ti6Al-4V specimens built by electron beam melting ( EBM ) and selective laser melting ( SLM ), Materials Characterization 143 (November 2017) (2018) 76-81. doi:10.1016/j .matchar.2018.03.028. 
[9] H. K. Rafi, N. V. Karthik, H. Gong, T. L. Starr, B. E. Stucker, Microstructures and mechanical properties of Ti6Al4V parts fabricated by selective laser melting and electron beam melting, Journal of Materials Engineering and Performance 22 (12) (2013) 3872-3883. arXiv:arXiv:1011.1669v3, doi:10.1007/ s11665-013-0658-0.

[10] N. Hrabe, T. Gnäupel-Herold, T. Quinn, Fatigue properties of a titanium alloy (Ti-6Al-4V) fabricated via electron beam melting (EBM): Effects of internal defects and residual stress, International Journal of Fatigue 94 (2017) 202-210. doi:10.1016/j.ijfatigue.2016.04.022.

[11] S. J. Li, Q. S. Xu, Z. Wang, W. T. Hou, Y. L. Hao, R. Yang, L. E. Murr, Influence of cell shape on mechanical properties of Ti- $6 \mathrm{Al}-4 \mathrm{~V}$ meshes fabricated by electron beam melting method, Acta Biomaterialia 10 (10) (2014) 4537-4547. doi:10.1016/j .actbio.2014.06.010.

[12] V. Crupi, E. Kara, G. Epasto, E. Guglielmino, H. Aykul, Static behavior of lattice structures produced via direct metal laser sintering technology, Materials and Design 135 (2017) 246-256. doi:10.1016/j. matdes.2017.09.003.

[13] S. Y. Choy, C. N. Sun, K. F. Leong, J. Wei, Compressive properties of functionally graded lattice structures manufactured by selective laser melting, Materials and Design 131 (May) (2017) 112-120. doi: $10.1016 / j$.matdes . 2017.06.006.

[14] N. W. Hrabe, P. Heinl, B. Flinn, C. Körner, R. K. Bordia, Compression-compression fatigue of selective electron beam melted cellular titanium (Ti-6Al-4V), Journal of Biomedical Materials Research - Part B Applied Biomaterials 99 B (2) (2011) 313-320. doi:10.1002/jbm.b.31901.

[15] S. J. Li, L. E. Murr, X. Y. Cheng, Z. B. Zhang, Y. L. Hao, R. Yang, F. Medina, R. B. Wicker, Compression fatigue behavior of Ti-6Al-4V mesh arrays fabricated by electron beam melting, Acta Materialia 60 (3) (2012) 793-802. doi:10.1016/j.actamat.2011.10.051.

[16] S. M. Ahmadi, R. Hedayati, Y. Li, K. Lietaert, N. Tümer, A. Fatemi, C. D. Rans, B. Pouran, H. Weinans, A. A. Zadpoor, Fatigue performance of additively manufactured meta-biomaterials: The effects of topology and material type, Acta Biomaterialia (2017) 1-13doi:10.1016/j.actbio.2017.11.014.

[17] A. A. Zadpoor, Mechanical performance of additively manufactured meta-biomaterials, Acta Biomaterialia 85 (2019) 41-59. doi:10.1016/j.actbio.2018.12.038.

[18] M.-S. Pham, C. Liu, I. Todd, J. Lertthanasarn, Damage-tolerant architected materials inspired by crystal microstructure, Nature 565 (7739) (2019) 305.

[19] P. Köhnen, C. Haase, J. Bültmann, S. Ziegler, J. H. Schleifenbaum, W. Bleck, Mechanical properties and deformation behavior of additively manufactured lattice structures of stainless steel, Materials and Design 145 (2018) 205-217. doi:10.1016/j.matdes.2018.02.062. 
[20] L. Huynh, J. Rotella, M. D. Sangid, Fatigue behavior of IN718 microtrusses produced via additive manufacturing, Materials and Design 105 (2016) 278-289. arXiv:arXiv:1011.1669v3, doi:10.1016/ j.matdes.2016.05.032.

URL http://dx.doi.org/10.1016/j.matdes.2016.05.032

[21] F. Brenne, T. Niendorf, Load distribution and damage evolution in bending and stretch dominated Ti-6Al-4V cellular structures processed by selective laser melting, International Journal of Fatigue 121 (November 2018) (2019) 219-228. doi:10.1016/j.ijfatigue.2018.12.017.

[22] K. Lietaert, A. Cutolo, D. Boey, B. Van Hooreweder, Fatigue life of additively manufactured Ti6Al4V scaffolds under tension-tension, tension-compression and compression-compression fatigue load, Scientific Reports 8 (1) (2018) 1-9. doi:10.1038/s41598-018-23414-2.

[23] B. Van Hooreweder, Y. Apers, K. Lietaert, J. P. Kruth, Improving the fatigue performance of porous metallic biomaterials produced by Selective Laser Melting, Acta Biomaterialia 47 (2017) 193-202. doi: $10.1016 / j$. actbio. 2016.10.005.

[24] S. Demiray, W. Becker, J. Hohe, Investigation of the fatigue behavior of open cell foams by a micromechanical 3-D model, Materials Science and Engineering A 504 (2009) 141-149. doi:10.1016/j.msea. 2008.10 .036

[25] R. Hedayati, H. Hosseini-toudeshky, M. Sadighi, M. Mohammadi-aghdam, A. A. Zadpoor, Computational prediction of the fatigue behavior of additively manufactured porous metallic biomaterials, International Journal of Fatigue 84 (2016) 67-79. doi:10.1016/j.ijfatigue.2015.11.017.

URL http://dx.doi.org/10.1016/j.ijfatigue.2015.11.017

[26] A. Zargarian, M. Esfahanian, J. Kadkhodapour, S. Ziaei-Rad, Numerical simulation of the fatigue behavior of additive manufactured titanium porous lattice structures, Materials Science and Engineering C 60 (2016) 339-347. doi:10.1016/j.msec.2015.11.054.

[27] A. Zargarian, M. Esfahanian, J. Kadkhodapour, S. Ziaei-rad, On the fatigue behavior of additive manufactured lattice structures, Theoretical and Applied Fracture Mechanics 100 (November 2018) (2019) 225-232. doi:10.1016/j.tafmec.2019.01.012.

URL https://doi.org/10.1016/j.tafmec.2019.01.012

[28] M. Miner, et al., Cumulative fatigue damage, Journal of applied mechanics 12 (3) (1945) A159-A164.

[29] E. Wycisk, A. Solbach, S. Siddique, D. Herzog, F. Walther, C. Emmelmann, Effects of defects in laser additive manufactured Ti-6Al-4V on fatigue properties, Physics Procedia 56 (C) (2014) 371-378. doi: $10.1016 / j$.phpro.2014.08.120.

[30] S. Tammas-Williams, P. J. Withers, I. Todd, P. B. Prangnell, The Influence of Porosity on Fatigue Crack Initiation in Additively Manufactured Titanium Components, Scientific Reports 7 (1) (2017) 1-13. doi:10.1038/s41598-017-06504-5. 
[31] T. Persenot, A. Burr, R. Dendievel, J.-Y. Buffière, E. Maire, J. Lachambre, G. Martin, Fatigue performances of chemically etched thin struts built by selective electron beam melting: experiments and predictions, Materialia (2020) 100589.

[32] T. Persenot, G. Martin, R. Dendievel, J. Y. Buffiére, E. Maire, Enhancing the tensile properties of EBM as-built thin parts: Effect of HIP and chemical etching, Materials Characterization 143 (2018) 82-93. doi:10.1016/j.matchar.2018.01.035.

[33] M. Suard, G. Martin, P. Lhuissier, R. Dendievel, F. Vignat, J. J. Blandin, F. Villeneuve, Mechanical equivalent diameter of single struts for the stiffness prediction of lattice structures produced by Electron Beam Melting, Additive Manufacturing 8 (2015) 124-131. doi:10.1016/j.addma.2015.10.002.

[34] M. Dumas, P. Terriault, V. Brailovski, Modelling and characterization of a porosity graded lattice structure for additively manufactured biomaterials, Materials and Design 121 (2017) 383-392. doi: $10.1016 / j$.matdes . 2017.02.021.

[35] M. Dallago, V. Fontanari, E. Torresani, M. Leoni, C. Pederzolli, C. Potrich, M. Benedetti, Fatigue and biological properties of Ti-6Al-4V ELI cellular structures with variously arranged cubic cells made by selective laser melting, Journal of the Mechanical Behavior of Biomedical Materials 78 (2018) 381-394.

[36] G. Savio, S. Rosso, A. Curtarello, R. Meneghello, Implications of modeling approaches on the fatigue behavior of cellular solids, Additive Manufacturing 25 (November 2018) (2019) 50-58. doi:10.1016/j . addma. 2018.10.047.

[37] H. Alsalla, L. Hao, C. Smith, Fracture toughness and tensile strength of 316L stainless steel cellular lattice structures manufactured using the selective laser melting technique, Materials Science and Engineering A 669 (2016) 1-6. doi:10.1016/j.msea.2016.05.075.

[38] R. M. Latture, R. X. Rodriguez, L. R. Holmes, F. W. Zok, Effects of nodal fillets and external boundaries on compressive response of an octet truss, Acta Materialia 149 (2018) 78-87. doi:10.1016/j.actamat. 2017.12 .060 .

[39] J. Schindelin, I. Arganda-carreras, E. Frise, V. Kaynig, T. Pietzsch, S. Preibisch, C. Rueden, S. Saalfeld, B. Schmid, J.-y. Tinevez, D. J. White, V. Hartenstein, P. Tomancak, A. Cardona, Fiji - an Open Source platform for biological image analysis, Nature Methods 9 (7) (2013). doi:10.1038/nmeth.2019.Fiji.

[40] CEA, Cast3m, http://www-cast3m.cea.fr/index.php (2018).

[41] O. H. Basquin, The exponential law of endurance tests, Proc. Am. Soc. Test. Mater. 10 (1910) 625-630.

[42] T. Persenot, J. Buffière, E. Maire, R. Dendievel, G. Martin, Fatigue properties of EBM as-built and chemically etched thin parts, in: Procedia Structural Integrity 7, 2017, pp. 158-165.

[43] T. Persenot, Fatigue of ti-6al-4v thin parts made by electron beam melting, Ph.D. thesis, Université de Lyon (2018). 
[44] W. Hwang, K. Han, Cumulative Damage Models and Multi-Stress Fatigue Life Prediction, Journal of Composite Materials 20 (March 1986) (1986) 125-153.

[45] S. Romano, S. Miccoli, S. Beretta, A new FE post-processor for probabilistic fatigue assessment in the presence of defects and its application to AM parts, International Journal of Fatigue 125 (February) (2019) 324-341. doi:10.1016/j.ijfatigue.2019.04.008.

URL https://doi.org/10.1016/j.ijfatigue.2019.04.008

[46] C. M. Portela, J. R. Greer, D. M. Kochmann, Impact of node geometry on the effective stiffness of non-slender three-dimensional truss lattice architectures, Extreme Mechanics Letters 22 (2018) 138-148. doi:10.1016/j.eml.2018.06.004.

[47] V. Deshpande, M. Ashby, N. Fleck, Foam topology: bending versus stretching dominated architectures, Acta materialia 49 (6) (2001) 1035-1040.

[48] Simulia, Abaqus/cae, https://www.3ds.com/products-services/simulia/products/abaqus/ abaquscae/ (2014). 